\title{
UML-based Integration Testing for Component-based Software
}

\author{
Ye $\mathrm{Wu}^{1}$ and Mei-Hwa Chen ${ }^{2}$ and Jeff Offutt ${ }^{1}$ \\ 1 Information and Software Engineering Department \\ George Mason University \\ Fairfax, VA 22030, USA \\ \{wuye, ofut\}@ise.gmu.edu \\ 2 Computer Science Department \\ State University of New York at Albany \\ Albany, NY 12222, USA \\ mhc@cs.albany.edu
}

\begin{abstract}
Component-based software engineering is increasingly being adopted for software development. Currently, components delivered by component providers only include specifications of the interfaces. This imposes significant difficulties on adequate testing of an integrated component-based system. Without source code, many testing techniques will not be applicable. UML, a modeling language, has been widely adopted in component-based software development process. Many of its useful tools, such as interaction diagrams, statechart diagrams, and component diagrams, characterize the behavior of a component in various aspect, and thus can be used in testing component-based systems. In this paper, we first analyze different test elements that are critical to test component-based software, Then we propose a group of UML-based test elements, test adequacy criteria to test component-based software.
\end{abstract}

Keywords Component-based software, software testing, program analysis

\section{Introduction}

In his survey, Allen predicted that by the year 2003, up to $70 \%$ of all new software-intensive systems will heavily rely on component-based software [2]. A component-based software system often consists of a set of self-contained and loosely coupled components that allow plug-and-play integration. The components may have been written in different programming languages, executed in various operational platforms, and distributed across vast geographic distances; some components may be developed in-house, while others may be third party or commercial off-the-shelf components(COTS), whose source code may not be available to developers. These component-based software characteristics may facilitate fast-paced delivery of scalable and evolvable software, as well as improve the quality of the final products. However, these characteristics also introduce new problems for testing component-based software systems [17]. 
This research assumes that individual components have been thoroughly tested by component providers. But when integrating them in a new context, unexpected result may occur [17]. Therefore, adequate integration of reusable components is the key to the success of a component-based software system. Test methodologies are generally categorized into two types: black box and white box. Black box approaches, such as functional testing and random testing, do not require knowledge of the implementation details. But when applied to component-based software, the use of black box approaches may encounter problems similar to those found in the testing of traditional programs, i.e. the complexity of the actual combination of functions presented in the real system. Thus, white box approaches are often used to complement functional testing to ensure the quality of the programs. However, component-based software has two properties, heterogeneity and implementation transparency (the implementation is not available), which together prevent traditional white-box techniques from being used directly to test component-based software.

To overcome these difficulties, we need to precisely represent the behavior of components without source code. The Unified Modeling Languages (UML) [5] is a language for specifying, constructing, visualizing, and documenting artifacts of software-intensive systems that can be used. There are several advantages to adopting the UML. First, the UML provides high level information that characterize the internal behavior of components, which can be processed efficiently and used effectively when testing. Second, the UML has emerged as the industry standard for software modeling notations and various diagrams are available from many component providers. Third, the UML includes a set of models that can provide different levels of capacity and accuracy for component modeling, and thus can be used to satisfy various needs in the real world. In the UML, collaboration diagrams and sequence diagrams are used to represent interactions among different objects in a component, which in this research is used to develop a corresponding interaction graph that will be used to evaluate the control flow of a component. Statechart diagrams, on the other hand, are used to characterize internal behaviors of objects in a component. Based on the statechart diagram, we further refine the dependence relationships among interfaces and operations that are derived from collaboration diagrams.

The remainder of this paper briefly describes some of the background about component-based engineering and software testing in Section 2. Section 3 provides a test model for component-based software, and various UML-based test

elements are described in section 4 . Related research in testing component-based software systems are discussed in section 5 , with conclusions in section 6 .

\section{Background}

The component-based software literature has introduced a number of new terms, some of which are still used inconsistently. This section of the paper defines these terms as used in this paper. 
There have been several definitions of software components. Szyperski and Pfister [7] provide the distinctive nature of components from a structural perspective: A component is "a unit of composition with contractually specified interfaces and explicit context dependencies only. A software component can be deployed independently and is subject to composition by third parties." Brown [4] defines a component in a broader aspect: A component is "an independently deliverable piece of functionality providing access to the services through interfaces."

Interfaces are the access points of components, through which a client component can request services declared in the interface and provided by another component. Each interface is identified by an interface name and a unique interface ID. Each interface can include multiple operations, where each operation performs one specific service. For clarity, we assume one interface only includes one operation, and the references to the interface and to the operation are identical.

We define a event as an incident in which an interface is invoked in response to the incident. We consider only external events in which the responding entity is external to the invoking entity. The incident may be triggered by a different interface, through an exception or simply through a user action such as pushing a button. Some exceptions and user actions that require other components to respond may not occur in any interface of a component. To simplify our discussion, we define a virtual interface to account for all these possible incidents. Therefore, in general, we define an event as an invocation of an interface through another interface.

\section{Methodology}

\subsection{A test model for component-based software}

When testing a component-based software system, we assume each individual component has been adequately tested. Therefore, ensuring the accuracy of interactions among the components is key to the success of a reliable software system.

Components may interact with other components either directly or indirectly. Direct interaction includes invocation of the interfaces exposed by the components, an exception, or a user action triggering an event. Indirect interaction is through a sequence of events. The following defines four key elements that model the characteristics of the interactions, which need to be taken into accounted in component-based testing.

Interfaces: Interfaces are the most common ways to activate components. Therefore, it is necessary during integration and system testing to test each interface in the integrated environment at least once.

Events: Testing interfaces provides confidence that every interface that is possibly invoked during run time has been exercised at least once. This scenario is similar to the traditional test criterion that requires every function or procedure 
to be tested at least once. However, an interface invoked by different components within different contexts may have different outcomes. Thus, to observe possible behaviors of each interface during runtime, every invocation of the interface needs to be tested at least once. Moreover, some events that are not triggered via interfaces may have an impact on the components, which need to be tested as well. Therefore, every event in the system regardless of its type needs to be covered by some test.

Context-dependence Relationships: Interface and event testing ensure that every interaction between two components, the client and the server, is exercised. However, when execution of a component-based software system involves interactions among a group of components, the sequence of event triggering may produce unexpected outcomes. To capture the inter-relationships among events, we define a context dependence relationship that is similar to the control flow dependence relationship in traditional programs. An event $e_{2}$ has a contextsensitive dependence relationship with event $e_{1}$ if there exists an execution path where triggering of $e_{1}$ will directly or indirectly trigger $e_{2}$. For a given event $e$, it is necessary to test $e$ with every event that has a context-sensitive dependence relationship with $e$. This allows the tester to observe the possible impact of execution history on the outcome of the execution of $e$.

Context-sensitive dependence relationships include not only direct interactions, but also the indirect collaboration relationships among interfaces and events occurring through other interfaces and events as well. Therefore, testing context-sensitive dependence relationships may serve to identify interoperability faults caused by improper interactions among different components.

Content-dependence Relationships: An invocation of an interface of a component is in fact an invocation of a function implemented by the component. Therefore, when a function declared in an interface $v_{1}$ has data dependence relationship with another function declared in another interface $v_{2}$, the order of invocation of $v_{1}$ and $v_{2}$ could impact the results. A content-dependence relationship exists between two interfaces $v_{1}$ and $v_{2}$ if the two interfaces have a data-dependence relationship. An interface encapsulates one or more signatures, where each signature is a declaration of a function. When an interface is invoked, one or more functions will be executed to perform the requested service. Thus, the interface dependence relationship can be derived from the function dependence relationship, which we have shown elsewhere to be useful information in object-oriented class testing [6]. More precisely, a function $f_{2}$ depends on a function $f_{1}$ if and only if the value of a variable defined in $f_{1}$ is used in $f_{2}$. Therefore, a content-dependence relationship is formally defined as follows: An interface $v_{2}$ has a content-dependence relationship on interface $v_{1}$ if and only if $v_{1}$ contains the signature of $f_{1}, v_{2}$ contains the signature of $f_{2}$ and $f_{2}$ depends on $f_{1}$.

Both the direct interaction among interfaces and events, as well as the contextdependence relationships, should be included in the control flow interactions of a component-based system. Content-sensitive dependence, on the other hand, can provide valuable additional information in generating test cases and detecting faults. 


\subsection{UML-based integration testing for component-based software}

The test model presented in the previous section has present an effective way for testing component-based software. However, the implementation of the model faces a technical challenge- how to efficiently obtain the test elements to perform testing, particularly when the source code of the components is not available for COT components. Without source code, we can obtain the specifications of interfaces and events, however, context-dependence relationships and content- dependence relationships would be difficult to derive without a sophisticate mechanism. These two elements are much more effective than others in detecting component integration faults. Therefore, it is important to develop a methodology to obtain these two elements from the available resources other than the source code. To this end, we utilize the Unified Modeling Languages (UML)[5, $11]$ as a tool for capturing component relationships.

In this section we describe how to use the UML diagrams to precisely derive context-dependence and content- dependence test elements. In the next section we explore some practical issues of implementing this test model.

Context-dependence relationships When integrating components, programmers typically focus on how to specify component interfaces and events. But how these interfaces and events will interact, and their potential risks to the integrated system, are usually not considered. Context-dependence relationships, which model how interfaces and events interact, can be derived through one of the following different approaches:

1. Collaboration/sequence diagram based approach.

UML collaboration diagrams and sequence diagrams focus on interactions among various objects within a use case (in our study, we refer to a use case as a component.) In UML sequence diagrams, interactions are ordered by time while in collaboration diagrams, the same information is presented with the interactions ordered in numeric message sequence order.

Figure 1 describes a partial collaboration diagram and one sequence diagram of an ATM server component. The sequence diagram only shows one of the possible scenarios ordered by time while the collaboration combines all scenarios in numbered order. In Figure 1, W5, W5A, and W5B demonstrate three alternatives that can occur after the message W4 is passed by the Withdrawal Transaction Manager object to the ATM Account object.

With the collaboration diagrams, we can refine our context-dependence relationships to be all possible sequences, as shown in Figure 1 in a collaboration diagram that could be possibly invoked to precisely model how interfaces and events interact with each other.

2. Statechart based approach.

To model behaviors of the interactions of components, the collaboration diagram itself is not always sufficient. The behavior of a component can be more precisely modeled by combining the collaboration diagram with statechart diagrams, which are used to describe state-dependent control objects in components. 


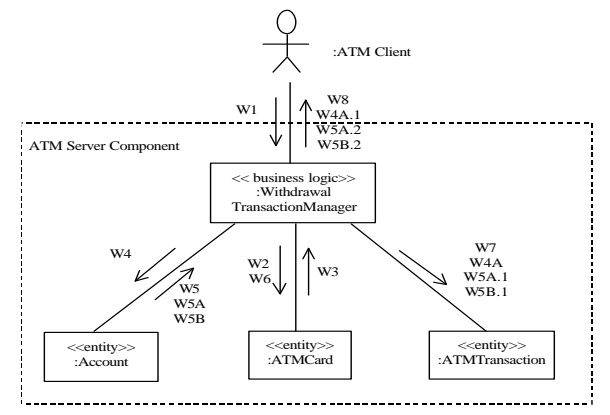

Collaboration Diagram

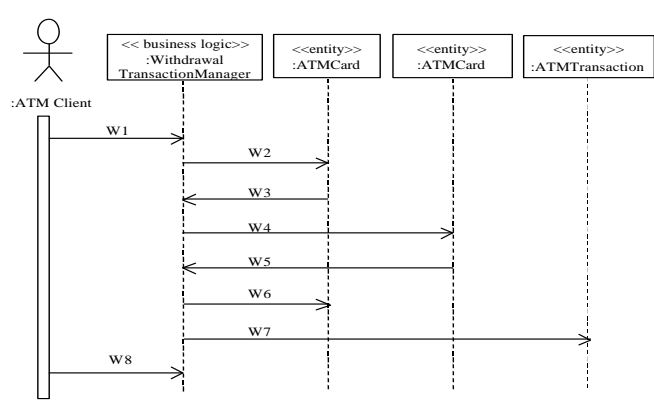

Sequence Diagram

\begin{tabular}{|ll|}
\hline W1: Withdrawal Request (Transaction Detail) & W5A.1: Log Transaction \\
W2: Check Daily Limit (Card ID, Amount) & W5A.2: Withdrawal Response (Invalid Account) \\
W3: Daily Limit Response & W5B: Insufficient Funds \\
W4: Debit (Account \#, Amount) & W5B.1: Log Transaction \\
W4A: Log Transaction & W5B: 2: Withdrawal Response (Insufficient funds) \\
W4A.1 : Withdrawal Response (Exceed Daily limit) & W6: Update Daily Limit (Card ID, Amount) \\
W5: Account Data & W7: Log Transaction \\
W5A: Invalid Account & W8: Withdrawal Response \\
\hline
\end{tabular}

Fig. 1. Collaboration and Sequence Diagrams of an ATM Server Component

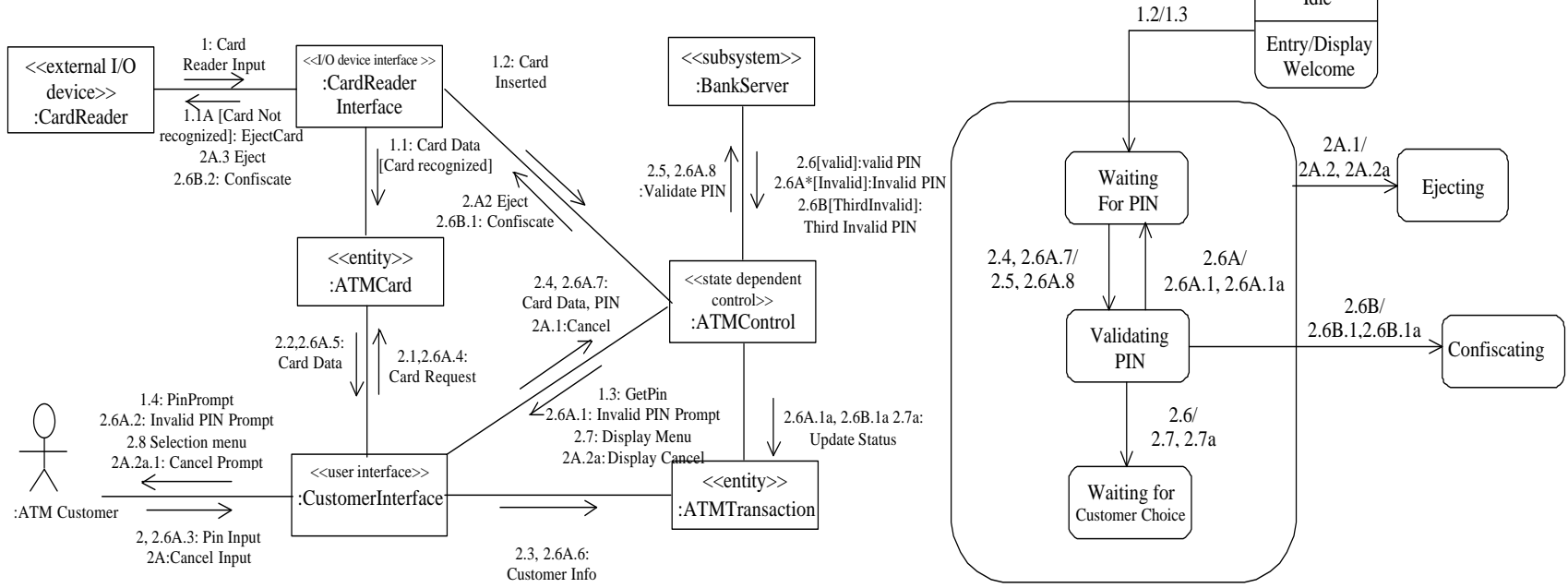

Fig. 2. Collaboration Diagram and Statechart for Validate PIN 
For example, Figure 2 shows a collaboration diagram and statechart. As we can see, the sequence "2A - 2A.1 - 2A.2, 2A.2a - 2A.3, 2A.2a.1" (2A.2 and 2A.2a are two concurrent messages) is the only sequence that allows the user to cancel. Nevertheless, this sequence can happen in different contexts, such as the user canceling the current transaction after correctly inputting the PIN, or the user canceling the current transaction after incorrectly inputting the PIN. With the help of the statechart diagram, which is shown in Figure 2(b), we can clearly see that the cancellation sequence needs to be validated in three different scenarios: (1) waiting for PIN, (2) validating PIN and (3) waiting for Customer Choice.

By using the Startchart diagram, the interactions among interfaces and events can be further refined. Given a startchart diagram, our context-dependence relationships will have to include not only all possible sequences in a collaboration diagram, but all possible combinations of the sequences that are shown in the startchart diagram as well.

Content-dependence relationships Context-dependence relationships reflect control sequences of objects in a component with respect to single interactions between actors and the component. Nevertheless, content-dependence relationships among different interactions cannot be obtained solely from control flow information. For example, consider Figure 3, which shows an extended ATM server component. The component includes two interfaces- withdraw and deposit. Context dependence relationships may depict the interactions within each of the interfaces, but the content-dependence relationships across the interfaces cannot be obtained. For instance, the withdrawal interface depends on deposit interface because deposit transaction will modify the account entity, while withdrawal transaction will use that entity to verify whether there is enough money in that account. Unfortunately, content-dependence relationships are not directly specified either in the program or in any UML diagrams. To specify the contentdependence relationships, further processing of UML diagrams is necessary. Several approaches can be adopted.

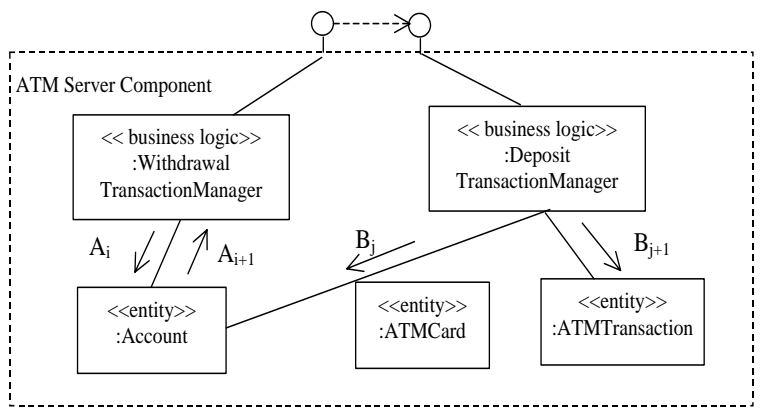

Fig. 3. An Extended ATM Server Component 
1. Collaboration diagram approach.

UML collaboration diagrams/sequence diagrams demonstrate interactions of objects within a component. When interactions involve entity classes, collaboration diagrams can demonstrate the dependence relationships between two interactions. For example, Figure 3 shows message $B_{j}$ flowing into entity class Account, and no information flows out of Account. Generally speaking, message $B_{j}$ will update information in Account objects. We define this type of messages to be update messages. On the contrary, messages $A_{i}$ and $A_{i+1}$ flow into and out of Account, which indicates that information of Account is retrieved. These types of messages are called retrieve messages. Therefore, interactions that include messages $A_{i}$ and $A_{i+1}$ will depend on sequences that includes message $B_{j}$. In general, an interface $I$ depends on interface $I^{\prime}$ if and only if a message sequence invoked by $I$ includes an update message, and another sequence invoked by $I^{\prime}$ includes a corresponding retrieve message.

2.Statechart diagram approach.

Statechart diagrams are able to demonstrate content dependence relationships from a state transition point of view. The rationale lies in the fact that if interface $I_{1}$ depends on $I_{2}$, the state of the component is $S_{1}$ after the execution of $I_{1}$. When executing interface $I_{2}$, the state transitions from $S_{1}$ to $S_{2}$ depend on state $S_{1}$ and the invocation $I_{1}$. To model this type of content dependence relationships, we eliminate dependence relations that are not effective:

- If the invocation of $I_{2}$ remains in the original state $S_{1}$ after the invocation of $I_{1}$, the dependence relationship does not affect the behavior of the software; the dependence relationship is not effective.

- From a certain state $S$, if the invocation of $I_{2}$, will always bring the state to $S^{\prime}$, it would not matter if interface $I_{1}$ is invoked before that or not. This indicates that the state transformation is not caused by the dependence relationships.

UML-based test adequacy criteria Given the UML-based context dependence relationships and content dependence relationships, test criteria that is provided in our test model has to be modified as the following:

1. Each transition in each collaboration diagram has to be tested at least once.

2. Each valid sequence in each collaboration diagram has to be tested at least once.

3. Each transition in each statechart diagram has to be tested at least once.

4. Each content-dependence relationship derived from each collaboration diagram has to be tested at least once.

5. Each effective content-dependence relationship derived from each statechart diagram has to be tested at least once.

\section{Related Work}

The research results in this paper follow a small but growing body of work in component-based software testing. Weyuker [16] developed a set of axioms 
to help determine test adequacy and used them to evaluate several programbased testing techniques. Perry and Kaiser [14] further applied these adequacy axioms to object-oriented software and suggested that in the presence of objectoriented features, in particular inheritance and multiple inheritance, subclasses and superclasses require special attention during testing. Stemming from these studies, we developed a class testing technique [6] for testing object-oriented classes and programs.

In component-based testing, Rosenblum [15] proposed a formal model for adequate testing of component-based software, in which a "C$C$-adequate" criterion is defined to determine the adequacy of a test set for a given component-based software system as well as for a single component. A number of concepts $[8,9,18]$ have been proposed to analyze the characteristics of component-based software and suggest issues of testing component-based systems. Harrold et al. [9] proposed a testing technique that is based on analysis of component-based systems from component-provider and component-user perspectives. The technique was adapted from an existing technique [10], which makes use of complete information from components for which source code is available and partial information from those for which source code is not available. They [13] further extended their work by proposing a framework that lets component providers prepare various types of metadata such as program slicing. The metadata then used to help test component-based systems. Ghosh and Mathur [8] discussed issues in testing distributed component-based systems and suggested an interface and exception coverage-based testing strategy.

The UML is increasingly being used to support the design of componentbased systems $[5,11]$. Some of the UML diagrams have also been used to automatically generate test cases. Offutt and Abdurazik first proposed a mechanism that adapted state specification-based test data generation criteria to generate test cases from UML statecharts [12]. They subsequently extended their work to generate tests from UML collaboration diagrams [1]. At the same time, Lionel Briand and Yvan Labiche proposed to utilize class diagrams, collaboration diagrams or OCL to derive test requirements[3].

\section{Conclusions and Future Work}

This paper has presented a new approach for testing component-based software. It uses different UML diagrams to precisely model the internal behavior of third party software components. When the source is not available (as is usually the case), these behavioral descriptions can be used as a basis for deriving tests that can help ensure the quality of the component-based system. Our on-going research directions on this topic are empirical studies of comparisons of the effectiveness of our approach with other approaches, the development of a tool to support automation of the technique, and enhancement of the technique for resolving problems caused by distributed characteristics such as synchronization. 


\section{References}

1. Aynur Abdurazik and Jeff Offutt. Using UML collaboration diagrams for static checking and test generation. In Third International Conference on the Unified Modeling Language (UML '00), pages 383-395, York, UK, October 2000.

2. Paul Allen. Component-based development for enterprise systems: Applying the SELECT Perspective. Cambridge, UK, New York:Cambridge University Press, 1998.

3. Lionel Briand and Yvan Labiche. A UML-based appproach to system testing. In Fourth International Conference on the Unified Modeling Language (UML '01), pages 194-208, Toronto, Canada, October 2001.

4. Alan W. Brwan. Background information on CBD. SIGPC, 18(1), August 1997.

5. John Cheesman and John Daniels. UML components : A simple process for specifying component-based software. Addison-Wesley, 2001.

6. M. Chen and M. Kao. Effect of class testing on the reliability of object-oriented programs. In Proceedings of the Eighth International Symposium on Software Reliability Engineering, May 1997.

7. Szyperski Clemens. Component Software: Beyond Object-oriented Programming. Addison-Wesley, 1998.

8. S. Ghosh and A.P. Mathur. Issues in testing distributed component-based systems. In First International ICSE Workshop on Testing Distributed Component-Based Systems, Los Angeles, 1999.

9. M.J. Harrold, D. Liang, and S. Sinha. An approach to analyzing and testing component-based systems. In First International ICSE Workshop on Testing Distributed Component-Based Systems, Los Angeles, 1999.

10. M.J. Harrold and G. Rothermel. Performing dataflow testing on classes. In Proceedings of the Second ACM SIGSOFT Symposium on Foundations of Software Engineering, pages 154-163, December 1994.

11. George Heineman and William Councill. Component-based software engineering : Putting the pieces together. Addison-Wesley, 2001.

12. Jeff Offutt and Aynur Abdurazik. Generating test from UML specifications. In Second International Conference on the Unified Modeling Language (UML '99), pages 416-429, Fort Collins, CO, October 1999. IEEE Computer Society Press.

13. Alessandro Orso, Mary Jean Harrold, and David Rosenblum. Component metadata for software engineering tasks. In Proceedings of the 2nd International Workshop on Engineering Distributed Objects (EDO 2000), pages 126-140, 2000.

14. D. E. Perry and G. E. Kaiser. Adequate testing and object-oriented programming. Journal of Object-Oriented Programming, Jan. 1990.

15. D.S. Rosenblum. Adequate testing of component-based software. Technical Report TR97-34, University of California at Irvine, 1997.

16. E.J Weyuker. Axiomatizing software test data adequacy. IEEE Transtractions on Software Engineering, SE-1215(12):1128-1138, December 1986.

17. E.J Weyuker. Testing component-based software:A cautionary tale. IEEE Software, 15(5):54-59, September/October 1998.

18. Ye Wu, Dai Pan, and Mei-Hwa Chen. Techniques for testing component-based software. In 7th IEEE International Conference on Engineering of Complex Computer Systems, pages 222-232, Skovde, Sweden, June 2001. 